\title{
ACOUSTIC INSULATION OF SINGLE PANEL WALLS PROVIDED BY ANALYTICAL EXPRESSIONS VERSUS THE MASS LAW
}

\author{
A. Tadeu and J. M. P. António \\ Department of Civil Engineering, University of Coimbra, 3030-290 Coimbra, Portugal. \\ E-mail: tadeu@dec.uc.pt
}

(Received 30 July 2001, and in final form 2 January 2002)

\begin{abstract}
Analytical solutions are presented for the calculation of the acoustic insulation provided by an infinite single panel wall when subjected to a spatially sinusoidal harmonic line load or a point pressure load (modelled as a superposition of spatially sinusoidal harmonic line loads). The method used does not entail limiting the thickness of the layer, as the Kirchhoff or Mindlin theory requires, and fully takes into account the coupling between the fluid (air) and the solid panel. All calculations are performed in the frequency domain. Time signatures are obtained by means of inverse Fourier transforms. Special attention is given to the limitations of the simplified models, which are not able to predict dips of insulation such as that due to the coincidence effect. It has been shown that, although time results may appear complicated, the arrival of various pulses at the receivers can be understood in terms of the travelling body pulses and guided waves. Simulated results have been computed for ceramic, concrete and glass walls of different thickness, when subjected to plane, linear and spherical waves. The insulation computed was found to be highly dependent on receiver position, given the interaction between the incident wave field and the directed reflected field on the wall, when the wall is struck by a cylindrical or a spherical pulse wave.
\end{abstract}

(C) 2002 Elsevier Science Ltd. All rights reserved.

\section{INTRODUCTION}

The transmission of sound energy through a separation element proceeds by the vibration of the element, with the mass and sound frequency being relevant variables. As the mass of the element increases, so does insulation, due to increasing forces of inertia. When the frequency of sound incident on an element that maintains the same mass is increased, the vibration power of the element decreases and greater dissipation of sound energy is observed, leading to a rise in acoustic insulation.

Besides these two variables, there are others that may affect the acoustic insulation of a separation element. These include the angle of incidence of the waves, the existence of weak points in the insulation, rigidity, damping of the element and, in the case of multiple elements, the number of panels and their individual characteristics and separation. In a real situation, the transmission of sound between two contiguous rooms depends not only on the separation elements and the connections between the surrounding elements, but also on the way in which propagation proceeds inside the emitting and receptor rooms. In this process, the vibration eigenmodes of the rooms excited determine the manner of propagation.

The mathematical description of the phenomena involved in acoustic insulation is thus very complex. Studies are usually conducted with variations in only a limited number of the 
variables in question $[1,2]$. This results in a set of simplified predictive insulation models such as the Law of Theoretic Mass.

Other simplified methods have been proposed for predicting the sound insulation conferred by a single panel above, below, and in the vicinity of the coincidence effect [3-5]. A combination of the prediction methods presented by Sewell [3], Sharp [4] and Cremer [5] was used by Callister et al. [6] to evaluate the sound transmission loss of a single-layer panel. Novikov [7] proposed a method for expressing the sound insulation of finite plates at low excitation frequencies as the mass law plus a correction coefficient.

Osipov et al. [8] used three simplified theoretical models to predict the airborne sound transmission of single partitions at low frequencies, namely an infinite plate model, a baffled plate model and a room-plate-room model. In their models, the walls are assumed to be thin compared with the bending wavelength, and harmonic pure bending wave motion is adopted according to Kirchhoff's theory. The results reveal that sound insulation at low frequencies depends both on the properties of the separating wall and on the geometry and the dimensions of the room-wall-room system.

The sound transmission between adjacent rooms has also been addressed, using different numerical techniques such as the statistical energy analysis method (SEA), the finite element method (FEM) and the boundary element method (BEM).

The SEA is particularly suitable for studying the sound transmission through large and complex structures, where more accurate models would not be practical. This method divides the acoustic system into diverse subsystems. It is assumed that the response of a subsystem is determined by its resonant modes. However, the SEA model is unreliable at low frequencies due to the statistical uncertainties that occur where there are few resonant modes in each of the subsystems. The finite element model is not affected by this limitation at low frequencies. Steel and Craik [9] used both the SEA and the FEM to compute the sound transmission between walls, and compare these results with measured data. Comparison of the results showed that the FEM can be used for determining the coupling between subsystems. The SEA model was later used by Hynnä et al. [10] to predict the structure-borne sound transmission in large welded ship's structures. In the numerical technique developed, the pre-processing programs used in the context of the FEM were applied to reduce the modelling work. Recently, Craik and Smith [11] used a SEA model to compute the sound transmission through double-leaf lightweight partitions. At low frequencies the wall is modelled as a single subsystem, while at higher frequencies the SEA model makes use of a number of interconnected subsystems.

The finite element methods have not been used very often to compute sound insulation, because of the high computation cost entailed. They have failed because the domain being analyzed has to be fully discretized, and very fine meshes are needed to solve excitations at high frequencies. The FEM has been used to study the effect of room dimension on the sound insulation of a separating wall at low frequencies [12]. Maluski and Gibbs used the FEM to predict the sound insulation between adjacent rooms at low frequency, and compared the results with experimental data [13]. Results showed that the sound insulation provided by a separating wall at low frequencies is strongly dependent on the modal characteristics of the sound field of both rooms of the partition.

Sgard et al. [14] computed the low-frequency diffuse field transmission loss through double-wall sound barriers with elastic porous linings (composed of a porous-elastic decoupling material sandwiched between an elastic skin and a septum), using a finite element model for the different layers of the sound barrier coupled to a variational boundary element method to account for fluid loading. The diffuse field is assumed to be a combination of uncorrelated freely propagating plane waves with equal amplitude, no two of which are travelling in the same direction. Recently, the BEM has been used by Tinnsten 
et al. [15] to compute the pressure and intensity amplitude for structures vibrating in open domains, analyzing a vibrating sphere and an engine transmission cover.

The present work describes analytical solutions for calculating the acoustic insulation provided by a single panel wall when subjected to a spatially sinusoidal harmonic line load or a point pressure load (modelled as a superposition of spatially sinusoidal harmonic line loads). The method used does not entail limiting the thickness of the layer, as the Kirchhoff or Mindlin theory requires, and fully takes into account the coupling between the fluid (air) and the solid panel. It adapts the analytical equations developed by the authors for the steady state response of a homogeneous three-dimensional half-space and layered acoustic and elastic formations subjected to spatially sinusoidal harmonic line loads $[16,17]$.

This paper first describes the simplified model predicting insulation in single elements, known as the Law of Theoretic Mass, or the Law of Theoretical Frequency. There follows a description of the procedure for computing the analytical acoustic insulation provided by a single panel when subjected to a sinusoidal line pressure load. The full set of expressions is compared with those provided by the BEM, for which a full discretization of the boundary interfaces is required. Finally, a number of applications are presented to illustrate how the analytical solutions presented compare with those provided by the simplified analytical model.

\section{SIMPLIFIED MODEL PREDICTING INSULATION IN SINGLE ELEMENTS}

If an infinite simple separation element is held to behave like a group of juxtaposed masses, having independent displacement, and null damping forces, the sound reduction index $(R)$ for plane wave incidence follows a law, known as the Law of Theoretic Mass, or the Law of Theoretical Frequency [18],

$$
R=10 \log \left[1+\left(\frac{\pi f M \cos \theta}{\rho_{f} \alpha_{f}}\right)^{2}\right] \mathrm{dB}
$$

where $f$ is the frequency $(\mathrm{Hz}), M$ is the mass per unit area of the panel $\left(\mathrm{kg} / \mathrm{m}^{2}\right), \theta$ is the angle of incidence, $\rho_{f}$ is the density of the air $\left(\approx 1.22 \mathrm{~kg} / \mathrm{m}^{3}\right)$, and $\alpha_{f}$ is the speed of sound in air (for $T=20^{\circ} \mathrm{C}, \alpha_{f} \cong 340 \mathrm{~m} / \mathrm{s}$ ). This equation predicts an increase in the sound reduction index of about $6 \mathrm{~dB}$ for each doubling of the mass per unit area or frequency, but it does not take into account the existence of a diffuse field. Different models have been proposed for random distribution of angles of incidence, but again they all lead to an increase in the sound reduction index of about $6 \mathrm{~dB}$ for each doubling of the mass per unit area and frequency. The simplified model given by equation (1) is used throughout this work, for comparison with the computed results. The element's rigidity and damping affect its dynamic behavior, which is not taken into account by the models described above, leading to local dips in sound insulation. These can be predicted for frequencies relative to the normal transversal vibration modes through flexion and due to the propagation of plane waves throughout the panel.

The propagation of plane waves along a panel is frequently analyzed, assuming the existence of an infinitely long thin plate, not taking the presence of fluid on the two faces of the panel into account. The mathematical development of these assumptions leads to the dispersion relation [19]

$$
c_{L}=\left(D \omega^{2} / \rho h\right)^{0 \cdot 25},
$$


where $c_{L}$ is the phase velocity of the propagating plane waves along the plate, $\rho$ is the density of the material $\left(\mathrm{kg} / \mathrm{m}^{3}\right), h$ is the thickness of the panel $(\mathrm{m}), \omega=2 \pi f$, $D=h^{3} E /\left[12\left(1-v^{2}\right)\right]$ with $E$ and $v$, being Young's modulus and the Poisson ratio respectively. It should be noted that this equation predicts unbounded wave velocity for very short wavelength, high-frequency conditions, as a consequence of an imperfect mathematical model in which effects of shear and rotary inertia have been ignored. It is frequently accepted in practical applied acoustics that when the wavelength of sound air projected on a plate equals the wavelength of these bending waves, the movement of the panel increases, leading to a low sound insulation. This happens when

$$
\omega=\left(\alpha_{f} / \sin \phi\right)^{2} \sqrt{\rho h / D}
$$

where $\phi$ is the incidence angle of the sound relative to a direction perpendicular to the element. This assumption does not introduce significant misinterpretations in the insulation predictions because the sound waves travel at a much lower velocity than the body wave velocities in the plate.

The critical frequency $\left(f_{c}\right)$ is taken as being that which corresponds to $\phi=90^{\circ}$,

$$
f_{c}=\frac{\alpha_{f}^{2}}{1 \cdot 8138 h} \sqrt{\frac{\rho\left(1-v^{2}\right)}{E}} .
$$

\section{ANALYTICAL ACOUSTIC INSULATION PROVIDED BY A SINGLE PANEL}

This section briefly describes how the acoustic insulation provided by an elastic solid medium bounded by two acoustic flat fluid media, when subjected to a spatially sinusoidal harmonic pressure load, is analytically evaluated (see Figure 1(b)).

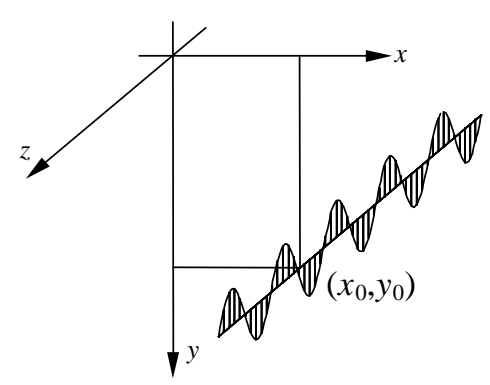

(a)

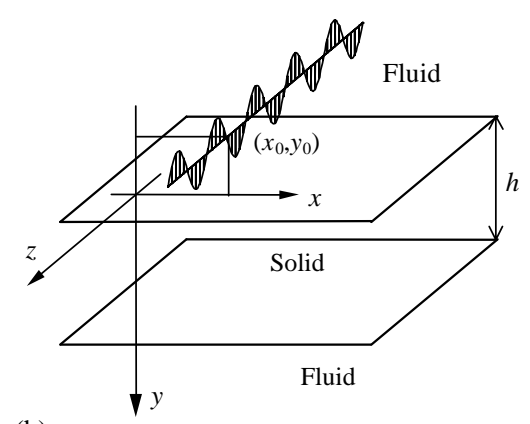

(b)

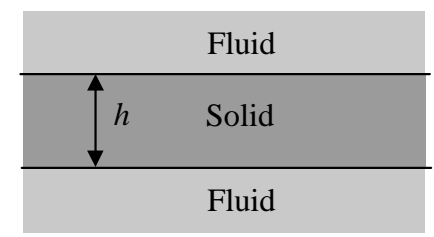

(c)

Figure 1. Definition of the problem: (a) spatially harmonic varying line load in an unbounded medium; (b) spatially sinusoidal harmonic line pressure load applied in the top fluid medium of a single panel structure bounded by two fluid parallel media; (c) geometry of the model. 
The solution uses the authors' previous work on deriving analytical solutions for the steady state response of a homogeneous three-dimensional half-space and layered acoustic and elastic formations subjected to spatially sinusoidal harmonic line loads $[16,17]$. The technique requires knowing the solid displacement potentials and fluid pressure potentials. The solid displacement potentials used to define the present Green functions are those defined in the method used by the authors [20] to evaluate the Green functions for a harmonic (steady state) line load with a sinusoidally varying amplitude in the third dimension, in an unbounded medium. These are in complete agreement with the solution for moving loads given earlier by Pedersen et al. [21] and Papageorgiou et al. [22]. A similar technique is employed for the fluid pressure potential. The displacement and pressure potentials are then written as a superposition of plane waves, following the approach used first by Lamb [23] for the two-dimensional case, and then by Bouchon [24] and Kim et al. [25] to calculate the three-space dimension field by means of a discrete wavenumber representation. The transformation of these integrals into a summation can be achieved if an infinite number of such sources are distributed along the $x$ direction, at equal intervals $L_{x}$ (see Appendix A).

This procedure yields the following expressions for the pressure field in the two fluid media, when a spatially sinusoidal harmonic pressure load is applied in the top layer of the fluid (see Figures 1(b) and 1(c)), at point $\left(x_{0}, y_{0}\right)$, in the vicinity of a solid layer of thickness $h$ :

$$
\begin{gathered}
\sigma^{f s f_{-} \text {top }}=\sigma^{f u l l}-\frac{\mathrm{i}}{L_{x}} \sum_{n=-N}^{n=+N}\left[\frac{E_{f 0}}{v_{n}^{f}} D_{n}^{f}\right] E_{d} \quad(\text { when } y<0), \\
\sigma^{f s f_{-} \text {bottom }}=-\frac{\mathrm{i}}{L_{x}} \sum_{n=-N}^{n=+N}\left[\frac{E_{f 0}^{b}}{v_{n}^{f}} H_{n}^{f}\right] E_{d} \quad(\text { when } y>h)
\end{gathered}
$$

where $E_{d}=\mathrm{e}^{-\mathrm{i} k_{n}\left(x-x_{0}\right)}, k_{n}=(2 \pi) /\left(L_{x}\right) n, v_{n}^{f}=\sqrt{k_{p_{f}}^{2}-k_{z}^{2}-k_{n}^{2}}$ with $\operatorname{Im}\left(v_{n}^{f}\right) \leqslant 0, k_{p_{f}}=\omega / \alpha_{f}$, $\omega$, is the frequency of the load, $k_{z}$ is the wavenumber in $z, \alpha_{f}=\sqrt{\lambda_{f} / \rho_{f}}$ is the acoustic (dilatational) wave velocity of the fluid, $\lambda_{f}$ is the fluid Lamé constant, $\rho_{f}$ is the mass density of the fluid, $E_{f 0}=\mathrm{e}^{-\mathrm{i} v_{n}^{f} y}, E_{f 0}^{b}=\mathrm{e}^{-\mathrm{i} v_{n}^{f}|y-h|}$ and $\sigma^{f u l l}$ is the direct incident pressure field (computed as in unbounded space). The coefficients $D_{n}^{f}$ and $H_{n}^{f}$ are determined from the appropriate boundary conditions $\left(\sigma_{y x}^{s}=\sigma_{y x}^{f}=0, \sigma_{y z}^{s}=\sigma_{y z}^{f}=0, \sigma_{y y}^{s}=\sigma_{y y}^{f}\right.$ and $u_{y}^{s}=u_{y}^{f}$ at $y=0$ and at $y=h$ ), so that the field produced simultaneously by the source and surface terms should produce the continuity of normal displacements and stresses, and null tangential stresses at the interface between the solid and the fluid media. The imposition of these boundary conditions, for each value of $n$ yields a system of eight equations in the eight unknown constants (see Appendix B). Finally, the acoustic insulation is given by the difference between $\sigma^{f s f_{-} t o p}$ and $\sigma^{f s f_{-} \text {bottom }}$, which can in turn be approximated by a finite sum of equations $(N)$.

\section{NUMERICAL APPLICATIONS}

The expressions described above were used to compute the acoustic insulation provided by a single infinite wall subjected to plane loads, spatially sinusoidal harmonic line loads or point loads. The plane load is simulated ascribing single values of $n$ and $k_{z}$ in equations (5), while the spatially sinusoidal harmonic line load is computed performing the full summation in $k_{n}$, again for a single value of $k_{z}$, using the same equations. The point load is 
TABLE 1

Material properties

\begin{tabular}{lcccccccccc}
\hline & $\begin{array}{c}\text { Shear } \\
\text { modulus } \\
(\mathrm{GPa})\end{array}$ & $\begin{array}{c}\text { Modulus } \\
\text { of } \\
\text { elasticity } \\
(\mathrm{GPa})\end{array}$ & $\begin{array}{c}\text { The } \\
\text { Poisson } \\
\text { ratio }\end{array}$ & $\begin{array}{c}\text { Density } \\
\left(\mathrm{kg} / \mathrm{m}^{3}\right)\end{array}$ & $\alpha(\mathrm{m} / \mathrm{s})$ & $\beta(\mathrm{m} / \mathrm{s})$ & Wall thickness $(\mathrm{cm})$ \\
\hline $\begin{array}{l}\text { Material } \\
\text { Concrete }\end{array}$ & $12 \cdot 6$ & $28 \cdot 98$ & $0 \cdot 15$ & 2500 & $3498 \cdot 6$ & 2245 & 5 & 10 & 15 & 20 \\
brick & $2 \cdot 5$ & $6 \cdot 0$ & $0 \cdot 20$ & 1400 & $2182 \cdot 2$ & $1336 \cdot 3$ & 5 & 10 & 15 & 20 \\
Glass & $29 \cdot 508$ & 72 & $0 \cdot 22$ & 2500 & $5734 \cdot 1$ & $3435 \cdot 6$ & $0 \cdot 4$ & $0 \cdot 8$ & $1 \cdot 2$ & $1 \cdot 6$ \\
\hline
\end{tabular}

synthesized by performing an inverse spatial Fourier transform in the $z$ direction, which is discretized, assuming the existence of an infinite number of equally spaced virtual sources.

The calculations are first performed in the frequency domain, which allows the insulation to be determined. Time responses are computed by means of inverse Fourier transforms. The source is assumed to be a Ricker wavelet function defined in the frequency domain by

$$
U(\omega)=A\left[2 \sqrt{\pi} t_{0} \mathrm{e}^{-\mathrm{i} \omega t_{s}}\right] \Omega^{2} \mathrm{e}^{-\Omega^{2}}
$$

where $A$ is the amplitude, $\Omega=\omega t_{0} / 2, t_{s}$ is the time when the maximum occurs, while $\pi t_{0}$ is the characteristic (dominant) period of the wavelet.

Thus, the Fourier transformations are achieved by discrete summations over wavenumbers and frequencies, which is mathematically the same as adding periodic sources at spatial intervals $L=2 \pi / \Delta k_{z}$ (in the $z$-axis), and $L_{x}=2 \pi / \Delta k_{n}$ (in the $x$-axis), and temporal intervals $T=2 \pi / \Delta \omega$, with $\Delta k_{z}, \Delta k_{n}$, being the wavenumbers and $\Delta \omega$ the frequency steps [26]. The spatial separations $L$ and $L_{x}$ must be large enough so that the periodic sources do not contaminate the response. Thus, the contribution to the response by the fictitious sources must occur at times later than $T$. This process is greatly helped if the frequency axis is shifted slightly downward, by considering complex frequencies with a small imaginary part of the form $\omega_{c}=\omega-\mathrm{i} \eta$ (with $\eta=0 \cdot 7 \Delta \omega$ ). The periodic sources are thus practically eliminated. In the time domain, this shift is later taken into account by applying an exponential window $\mathrm{e}^{\eta t}$ to the response [27].

In our examples, different materials and thickness $(h)$ were ascribed to the wall, as listed in Table 1. The host acoustic medium is kept constant, allowing a pressure wave speed of $340 \mathrm{~m} / \mathrm{s}$ and a density of $1.22 \mathrm{~kg} / \mathrm{m}^{3}$. The source is placed $2.0 \mathrm{~m}$ away from the wall, while a grid of receivers is placed on both sides of the wall, as shown in Figure 2.

The computations are performed in the frequency range $(2,8192 \mathrm{~Hz})$, with a frequency increment of $2 \mathrm{~Hz}$, which determines the total time duration $(T=0.5 \mathrm{~s})$ for the analysis in the time domain. The spatial distance between the virtual point sources has been set to $4 \alpha T$.

A selection of results is given below, to illustrate the main findings. First, a ceramic brick wall is used to illustrate how the acoustic insulation changes when subjected to plane waves with different incidence angles. Then, the time responses and insulation features are described, again using a ceramic brick wall subjected to the incidence of cylindrical waves $\left(k_{z}=0\right)$. Insulation curves for different wall materials and thickness are also included. The ceramic wall is used to show how the acoustic insulation varies when a panel is subjected to the incidence of cylindrical waves with different spatial sinusoidal variation along one 


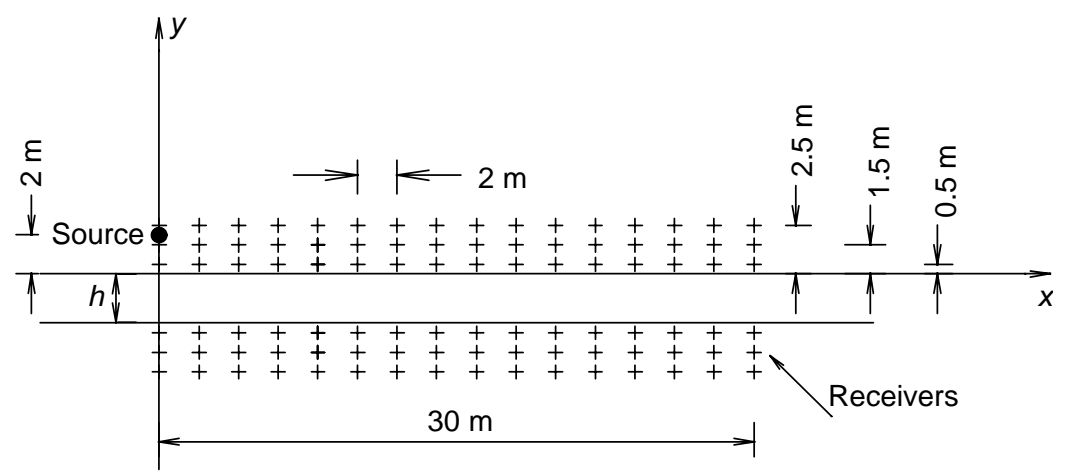

Figure 2. Position of the receivers and sources.

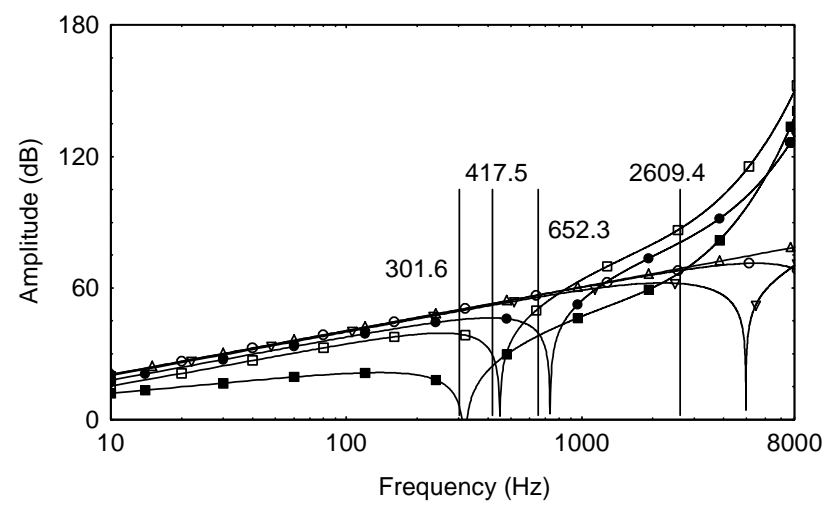

Figure 3. Sound transmission loss provided by a $10 \cdot 0 \mathrm{~cm}$ thick ceramic brick wall when subjected to plane waves $(\triangle$, Mass law; $\mathbf{\square}, v=340.0 \mathrm{~m} / \mathrm{s} ; \square, v=400.0 \mathrm{~m} / \mathrm{s} ; \boldsymbol{\wp}, v=500 \cdot 0 \mathrm{~m} / \mathrm{s} ; \nabla=1000 \cdot 0 \mathrm{~m} / \mathrm{s} ; \bigcirc, v=\infty \mathrm{m} / \mathrm{s})$.

direction $\left(k_{z} \neq 0\right)$. The last example provides the insulation response of a ceramic brick wall subjected to a point source.

\subsection{INCIDENCE OF PLANE WAVES}

Figure 3 illustrates the sound transmission loss provided by a ceramic brick wall, $10 \cdot 0 \mathrm{~cm}$ thick, in a dB scale, when subjected to plane waves with differing incidence angles, given by different apparent wave velocities, namely $v=\infty, 1000 \cdot 0,500 \cdot 0,400 \cdot 0$, and $340 \cdot 0 \mathrm{~m} / \mathrm{s}$. These computations have been performed ascribing $k_{z}=0$ in equations (5), and defining $N$ in these equations in such away as to model the different plane waves. Thus, $\mathrm{v}=\infty \mathrm{m} / \mathrm{s}$ $(N=0)$ corresponds to waves that are normally incident, while $v=340 \cdot 0 \mathrm{~m} / \mathrm{s}$ corresponds to waves that travel parallel to the ceramic wall. The waves travelling along the plate with apparent wave speeds of $v=1000 \cdot 0,500 \cdot 0$ and $400 \cdot 0 \mathrm{~m} / \mathrm{s}$ correspond to plane waves reaching the surface with inclinations of $19 \cdot 9^{\circ}, 42 \cdot 8^{\circ}$ and $58 \cdot 2^{\circ}$, respectively, in relation to the normal direction of the wall.

An additional curve, representing the mass law, given by equation (1), is also plotted in Figure 3. The comparison of the analytical curves with the mass law curve reveals that the 
analytical results differ from those provided by the mass law, unless the plane wave is normal to the wall $\left(v=\infty \mathrm{m} / \mathrm{s}\right.$ and $\left.k_{z}=0\right)$. In this case there is agreement, except for high frequencies. The other curves have dips at specific frequencies. When the apparent speed is taken to be equal to the sound speed in the air $(340 \cdot 0 \mathrm{~m} / \mathrm{s})$ the dip appears near to the critical frequency of the wall $\left(f_{c}=301.6 \mathrm{~Hz}\right)$, given by equation (4). However, the differences between the results computed by equation (3), for the frequencies associated with the plane waves travelling along the plate at speeds of $v=400 \cdot 0,500 \cdot 0$ and $1000 \cdot 0 \mathrm{~m} / \mathrm{s},(f=417 \cdot 5$, $f=652.3$ and $2609 \cdot 4 \mathrm{~Hz}$ ), and those of the insulation dips provided by our model, grows as the inclination of the waves decreases. Furthermore, as the apparent wave speed decreases, the insulation provided by the wall is observed to increase for high frequencies, whereas it decreases for low frequencies.

\subsection{INCIDENCE OF CYLINDRICAL WAVES}

Next, the wall is assumed to be subjected to the incidence of cylindrical waves of $k_{z}=0$. This corresponds to waves arriving at the receivers with a $90^{\circ}$ inclination in relation to the $z$-axis, which can be understood as a pure two-dimensional problem where the source is linear.

\subsubsection{Time responses}

Synthetic waveforms have been computed to simulate the wave propagation in the vicinity of a ceramic brick wall, $10 \cdot 0 \mathrm{~cm}$ thick. The response is computed at receivers placed $0.5 \mathrm{~m}$ away from the wall surface, on both sides of the wall.

Figure 4 displays the pressure time responses, modelling the result of the incidence of a cylindrical dilatational Ricker pulse source with a characteristic frequency $3000 \mathrm{~Hz}$ on the wall. Figure 4(a) illustrates the response obtained in the fluid medium containing the source. Two pulses are clearly visible, the direct incident pulse, labelled $I$ in the plot, and a pulse that arrives at the receivers after being reflected on the surface wall, labelled $P P$. However, the responses contain additional pulses, produced by refractions and mode conversions at the wall interface, labelled PPP and PSP waves. The PPP wave begins as a dilatational wave in the fluid; it is critically refracted onto the wall as a $P$ wave and then refracted back into the fluid as a dilatational wave. The so-called PSP wave begins as a dilatational wave in the fluid, it is critically refracted into the wall as an $S$ wave, and is refracted into the fluid as a dilatational wave. After the PSP wave arrives, the response is marked by a dense pack of pulses originating in the guided waves that travel along the wall. The scaling of the plots shown in Figure 4(a) does not allow easy observation of the PPP and PSP body waves. Since the scale of the plots precludes identification of the PPP and PSP body waves, we have amplified the graph so as to show these arrivals at the receiver placed at $x=28.0 \mathrm{~m}$ (see Figure 4(b)).

Figure 4(c) shows the synthetic signals computed in the fluid medium on the other side of the wall. As expected, the response amplitudes are much lower than the ones calculated for the side where the source is, due to the insulation provided by the wall. As before, a pack of high-frequency pulses is visible after the arrival of the dilatational waves that are caused by the incident waves critically refracting into the wall as $S$ waves, followed by a ring of low-frequency waves, up to the arrival of the later $P$ body pulses. Analysis of the results shows that the receiver, placed at the same abscissa as the source $(x=0.0 \mathrm{~m})$, does not record the presence of the guided waves. The guided waves are dispersive and originate at some distance from the source. 

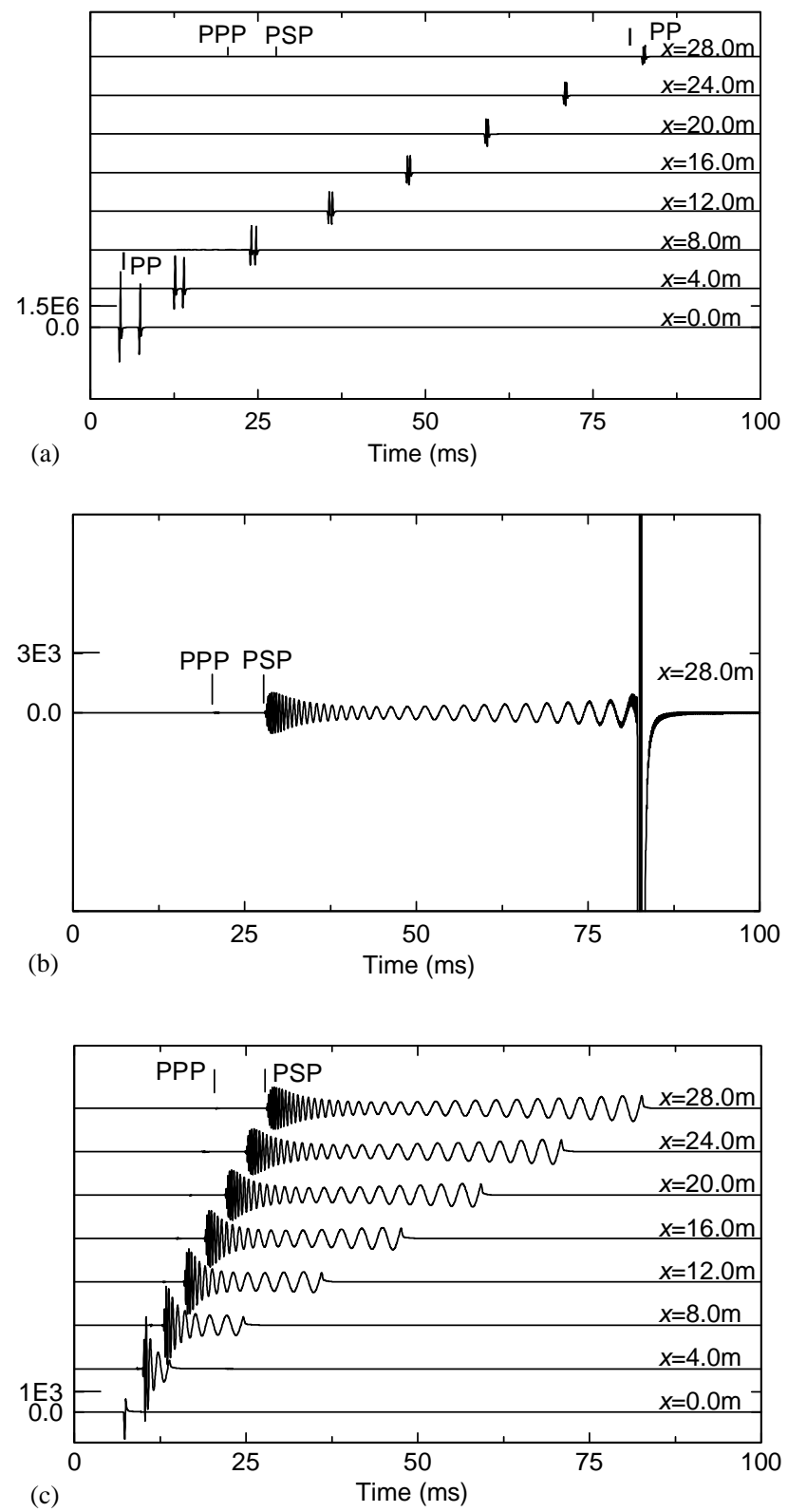

Figure 4. Synthetic waveforms at receivers placed $0.5 \mathrm{~m}$ away from a $10 \cdot 0 \mathrm{~cm}$ thick ceramic brick wall, subjected to a cylindrical dilatational Ricker pulse source with a characteristic frequency $3000 \mathrm{~Hz}\left(k_{z}=0\right)$ : (a) in the fluid medium containing the source. (b) in the fluid medium containing the source at $x=28 \cdot 0 \mathrm{~m}$; (c) in the fluid medium not containing the source.

To illustrate the behavior of the propagation at low frequencies, Figure 5 includes the responses obtained when the source excites a pulse with a frequency of $500 \mathrm{~Hz}$, which exhibits longer time duration. Figure 5(a) displays the results computed in the fluid medium containing the source. The arrival of the incident and the directed reflected pulses coincides with the corresponding arrival times obtained when the frequency excitation of the source 

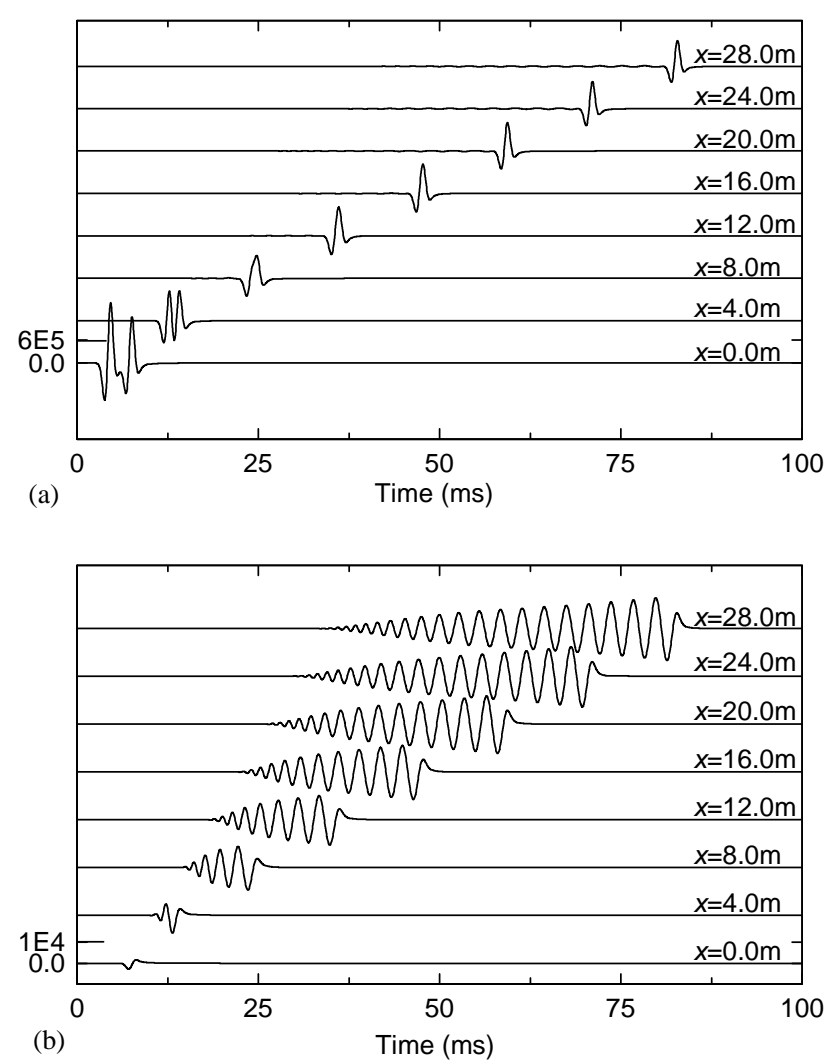

Figure 5. Time signals at receivers placed $0.5 \mathrm{~m}$ away from a $10.0 \mathrm{~cm}$ thick ceramic brick wall, subjected to a cylindrical dilatational Ricker pulse source with a characteristic frequency $500 \mathrm{~Hz}\left(k_{z}=0\right)$. (a) in the fluid medium containing the source; (b) in the fluid medium not containing the source.

was set to $3000 \mathrm{~Hz}$. At receivers placed further away from the source, however, a single pulse is detectable, caused by the incident pulse overlapping the directed reflected pulses.

Figure 5(b) shows the time responses calculated in the fluid medium on the other side of the source. The wave propagation features registered at Figure 4(c) are still visible. However, the guided waves arrive at later times, owing to the absence of the high frequency components, which exhibited faster velocities, as expected.

\subsubsection{Insulation calculations}

Next, a $10 \cdot 0 \mathrm{~cm}$ thick ceramic wall is used to illustrate some basic insulation features and show how the insulation is calculated. The insulation provided by the different wall types is then presented.

Figure 6 plots the amplitude of the responses, on a dB scale, on both sides of the ceramic wall at receivers placed at $x=28 \cdot 0,0 \cdot 5 \mathrm{~m}$ from the wall. The amplitude response on the side of the source (labelled top layer), evinces pronounced dips that result from the interaction of the incident wave field with the directly reflected field, which happens when the difference in travel paths $\Delta s$ is a multiple of the wavelength $\left(0 \cdot 5 \alpha_{f} / \Delta s=2386 \cdot 4 \mathrm{~Hz}\right.$ and $1 \cdot 5 \alpha_{f} / \Delta s=$ $7159 \cdot 3 \mathrm{~Hz}$ ). The amplitude response at the receiver on the other side of the wall (labelled "bottom layer"), is smoother but it shows an amplification at frequencies in the vicinity of 


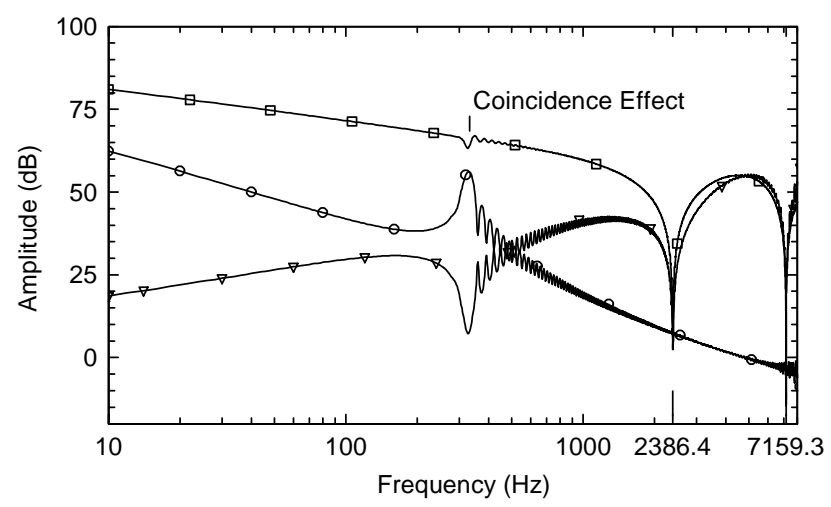

Figure 6. Amplitude of the responses on both sides of a $10 \cdot 0 \mathrm{~cm}$ thick ceramic wall at $x=28 \cdot 0,0 \cdot 5 \mathrm{~m}$, away from the wall, and computed insulation curve when subjected to a cylindrical two-dimensional line source ( $\nabla$, insulation; $\square$, top layer; $\bigcirc$, bottom layer).

the coincidence effect. This figure includes the insulation curve, provided by the difference between the amplitudes of the previous curves. In this curve, the dips observed for the receiver response placed in the side of the source are still evident, as is the coincidence effect observed at the receiver placed in the other side of the wall.

Figure 7(a) displays the insulation curves computed from the responses at receivers placed $0.5 \mathrm{~m}$ away from the wall, and at $x=0.0,8.0,16.0 \mathrm{~m}$ and $24.0 \mathrm{~m}$. The dips of insulation due to the interaction of the incident wave field with the directed reflected field of the different curves occurs at well-defined frequencies, as explained before. The insulation dip due to the coincidence effect is not observable at $x=0.0 \mathrm{~m}$. This was expected because the guided waves do not exist on the vertical plane containing the source, $x=0.0 \mathrm{~m}$. For the other curves, the coincidence effect is clearly visible and occurs in the vicinity of $\left(f_{c}=301.6 \mathrm{~Hz}\right)$, given by equation (4). Analysis of the results indicates that the insulation is highly dependent on the position of the receivers. Additional calculations have been performed to compute the average energy of the responses along the grid of receivers, illustrated in Figure 2, on both sides of the wall, for each frequency of excitation. Figure 7(b) shows the average insulation curve obtained by the difference between these two responses. The resulting curve is smoother, with a pronounced dip due to the coincidence effect.

Next, the average insulation curves computed for the various walls, with different thickness and materials, as listed in Table 1, are displayed in Figure 8. In each plot the insulation curve predicted by the mass law (equation (1)) for the thicker wall is also included, to illustrate the differences between the analytical model and the simplified model. The simplified curves for the other wall thickness are not plotted, to make it easier to interpret the results, but they would appear as parallel, decreasing $6 \mathrm{~dB}$ for each half of mass. Additional vertical lines have been positioned in the plots according to the critical frequency obtained by equation (4) for each wall.

The simplified model predicts an increase in sound insulation of about $6 \mathrm{~dB}$ for each doubling of frequency, which does not occur with the analytical solution. The increase of sound insulation with frequency is indeed less than predicted. At low frequencies the simplified model predicts insulation values lower than those obtained by the analytical model. It can also be seen that the discrepancies between the simplified insulation curves and analytical curves increase as the mass of the wall diminishes, that is, as the thickness decreases and with the change from the concrete to the ceramic wall and then to the glass wall. The position of the critical frequencies, predicted by the simplified model, equation (4), 

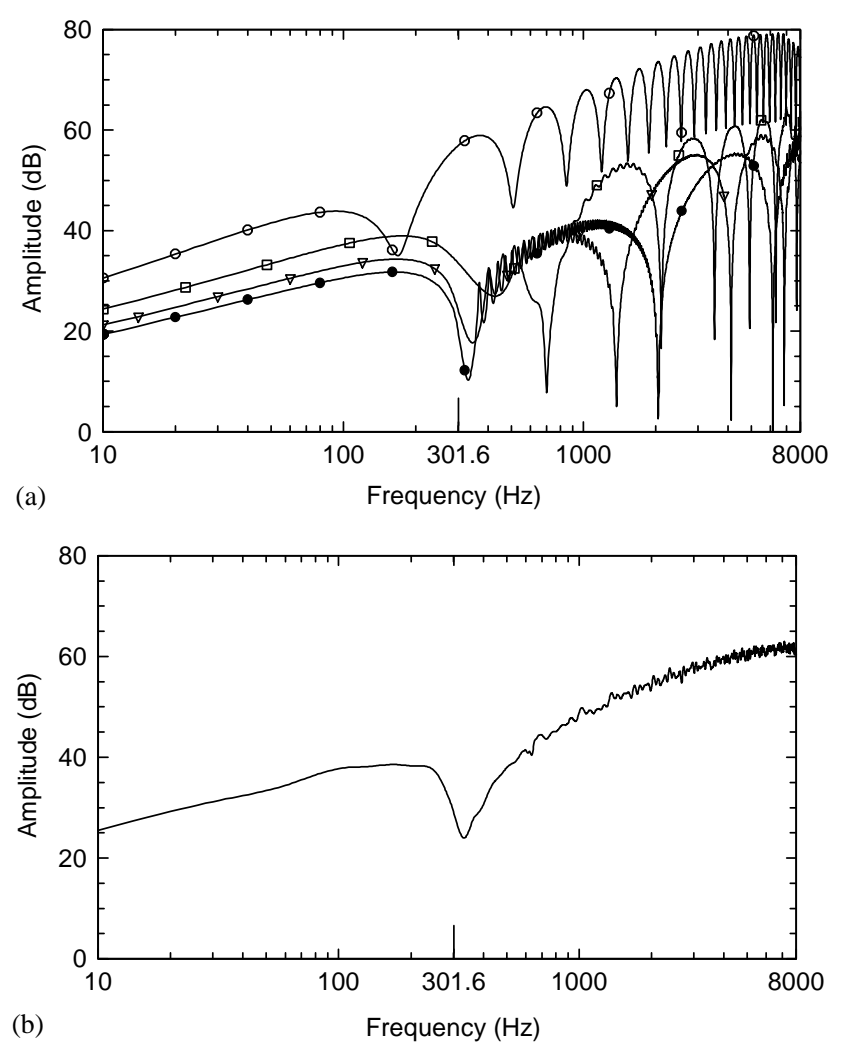

Figure 7. Computed insulation curves provided by a $10 \cdot 0 \mathrm{~cm}$ thick ceramic wall when subjected to a cylindrical line source $\left(k_{z}=0\right.$ ): (a) receivers placed $0.5 \mathrm{~m}$ away from the wall, at $x=0.0 \mathrm{~m}$ (marked $\bigcirc$ ), $x=8.0 \mathrm{~m}$ (marked $\square$ ), $x=16.0 \mathrm{~m}$ (marked $\nabla$ ) and $x=24.0 \mathrm{~m}$ (marked $)$; (b) average insulation obtained for the full grid of receivers.

is closer to the insulation dips calculated by the analytical model as we move from the ceramic to concrete wall, and then from the concrete to the glass wall, that is, as the modulus of elasticity of the material from which the wall is made increases.

A $10.0 \mathrm{~cm}$ thick ceramic wall is again used to illustrate how the acoustic insulation varies when a panel is subjected to the incidence of cylindrical waves with different spatial sinusoidal variation along one direction.

This type of load simulates the propagation of a moving load along the $z$ direction and simulations are performed, following waves with different apparent wave velocities along the $z$-axis. This apparent wave velocity $\left(v_{z}\right)$ results from waves arriving at the $z$ axis with a path inclination given by $\arccos \left(\alpha_{f} / v_{z}\right)$, where $\alpha_{f}$ is the true wave speed. Thus, in the equations presented above, $k_{z}$ is taken to be $\omega / v_{z}$. In the examples given, five apparent speeds $\left(v_{z}\right)$ are chosen, namely $v_{z}=\infty, 1000,500,400$ and $340 \mathrm{~m} / \mathrm{s}$. The apparent speed $v_{z}=\infty \mathrm{m} / \mathrm{s}$ corresponds to waves arriving at the receivers with a $90^{\circ}$ inclination in relation to the $z$-axis, $k_{z}=0$. As the path inclination ranges from $90^{\circ}$ to $0^{\circ}$, there is a lower bound value for $v_{z}$, which corresponds to the slowest wave speeds, $v_{z}=340 \mathrm{~m} / \mathrm{s}$. Below this value, there are inhomogeneous waves, which decay very quickly with decreasing values of $v_{z}$.

The average insulation curves computed for the different apparent wave velocities $\left(v_{z}\right)$ are displayed in Figure 9. The insulation curve predicted by the mass law (equation (1)) is also included. The insulation provided by the wall when struck by a linear source $\left(v_{z}=\infty \mathrm{m} / \mathrm{s}\right)$, exhibits a well-defined dip in the vicinity of the critical frequency given by 

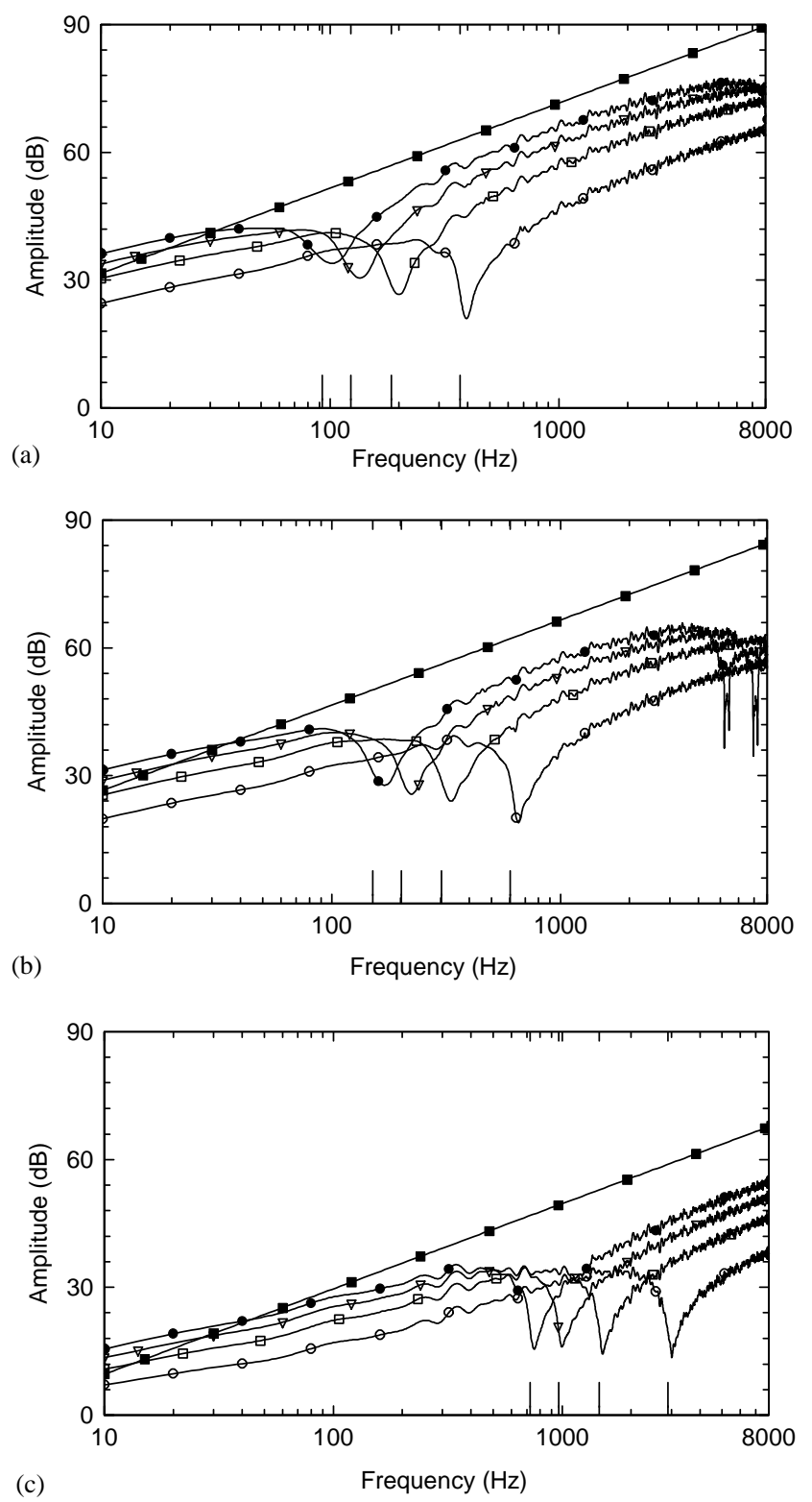

Figure 8. Computed average insulation curves provided by a wall subjected to a cylindrical line source $\left(k_{z}=0\right)$ : (a) concrete wall (ロ, Mass law; $, h=0.20 \mathrm{~m} \nabla, h=0.15 \mathrm{~m} ; \square, h=0.10 \mathrm{~m} ; \bigcirc, h=0.05 \mathrm{~m}$ ); (b) ceramic wall (घ, Mass law; $\bullet, h=0.20 \mathrm{~m} ; \nabla, h=0.15 \mathrm{~m} ; \square, h=0.10 \mathrm{~m} ; \bigcirc, h=0.05 \mathrm{~m}$ ); (c) glass wall (घ, Mass law; $h=0.016 \mathrm{~m} ; \nabla, h=0.012 \mathrm{~m} ; \square, h=0.008 \mathrm{~m} ; h=0.004 \mathrm{~m})$.

equation (4). As the apparent velocity diminishes, the insulation results include the presence of an additional dip, which appears at higher frequencies, caused by the different incident angles of the waves along the $z$ direction. The location of this second dip in the frequency domain coincides with that originated by plane waves illuminating the wall panel with an identical incidence angle along the $x$-axis (see Figure 3). When the apparent wave velocity reaches the sound wave speed $\left(v_{z}=340 \mathrm{~m} / \mathrm{s}\right)$ this second dip coincides with the critical frequency. 


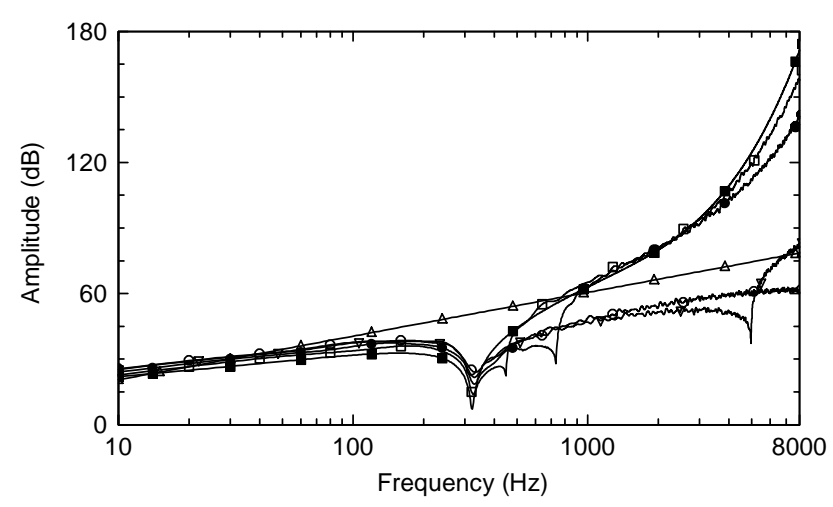

Figure 9. Average insulation curves computed when a $10 \cdot 0 \mathrm{~cm}$ thick ceramic wall is subjected to the incidence of cylindrical waves with different spatial sinusoidal variation along the $z$ direction $\left(\triangle\right.$, Mass law; $\mathbf{\square}, v_{z}=340 \cdot 0 \mathrm{~m} / \mathrm{s}$; $\left.\square, v_{z}=400 \cdot 0 \mathrm{~m} / \mathrm{s} ; 0, v_{z}=500 \cdot 0 \mathrm{~m} / \mathrm{s} ; v_{z}=1000 \cdot 0 \mathrm{~m} / \mathrm{s} ; \bigcirc, v_{z}=\infty \mathrm{m} / \mathrm{s}\right)$.

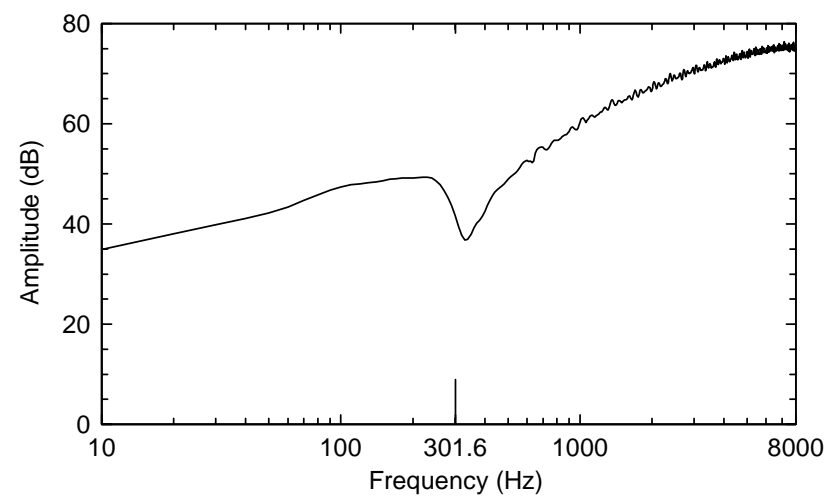

Figure 10. Average insulation curve computed when a $10 \cdot 0 \mathrm{~cm}$ thick ceramic wall, is subjected to the incidence of spherical wave.

The insulation obtained for cylindrical waves with different apparent wave speeds along the $z$ direction approaches the mass law prediction at low frequencies. In the vicinity of the coincidence effect, the computed insulation appears well below that given by the mass law. At high frequencies, lower insulation than that predicted by the mass law is calculated when the apparent wave speed, $v_{z}$, is high, while the computed insulation exceeds the prediction provided by the mass law when the apparent speed, $v_{z}$, is low.

\subsection{INCIDENCE OF SPHERICAL WAVES (POINT LOAD)}

The last example provides the insulation response of the $10 \cdot 0 \mathrm{~cm}$ thick brick wall, subjected to a point pressure source. The results have been computed, fully integrating the response in the $k_{z}$ wavenumber domain. Figure 10 displays the calculated average insulation results. Again, the dip of insulation related to the coincidence effect appears at the same frequency location as that for cylindrical waves $\left(k_{z}=0\right)$. The level of computed insulation is higher than before, particularly for high frequencies.

The time responses computed on both sides of the wall, when the source is modelled as a Ricker wavelet with a characteristic frequency of $3000 \mathrm{~Hz}$, manifest similar trends to the 
ones defined for a linear cylindrical source $\left(k_{z}=0\right)$. However, the amplitude of the signals decays faster than before $\left(k_{z}=0\right)$ as the receiver is placed further away from the source. This was anticipated, as the energy of a spherical wave decays faster with the distance to the source than that of a linear source (not displayed).

\section{CONCLUSIONS}

Analytical solutions for the steady state response of a spatially sinusoidal harmonic line load, illuminating a single panel wall, bounded by two flat fluid media, have been presented. These solutions make it possible to compute both the time pressure responses and the insulation provided by the solid wall, for plane, cylindrical and point pressure waves.

The synthetic signals computed in the fluid medium reveal the presence of a complicated wavefield, generated by both body waves and guided surface waves. The guided waves were found to be very dispersive, and faster phase velocities were associated with higher frequencies of excitation. At high frequencies of excitation, the arrival of the guided waves exhibits a pack of a high-frequency pulses, followed by a ring of low-frequency waves. The features of the time responses obtained when cylindrical waves are excited are similar to those obtained when a spherical pulse is excited. However, the amplitude decay of the response obtained when a spherical wave is excited is faster with the distance to the source than that of a linear source.

The results obtained when the wall is illuminated by plane waves indicate that the dips of insulation related to the coincidence effect occur in the vicinity of those computed using the dispersion relation for thin plates. However, the difference between the frequency positions of these dips, provided by the simplified model, and those computed by our model, grows as the incidence angle of the plane waves increases.

The computed insulation appears to be very dependent on receiver position, because of the interaction between the incident wave field and the field directly reflected on the wall, when the wall is struck by a cylindrical pulse wave. The average insulation calculated for the different wall materials and thickness is less than predicted by the simplified model, for frequencies above the coincidence effect. The computed insulation and the simplified results show more pronounced differences as the mass of the wall diminishes. The simplified determination of the critical frequencies approximates the insulation dips calculated by the analytical model more closely as the modulus of elasticity of the material wall increases.

The insulation results reveal the presence of an additional dip, which appears at higher frequencies, when the line source is sinusoidal along the $z$ direction. This second dip occurs at the same frequency as that originated by plane waves illuminating the wall panel with an identical incidence angle along the $x$-axis. When the wall is subject to a spherical pulse, the dip in insulation related to the coincidence effect occurs at the same frequency position as that for cylindrical waves $\left(k_{z}=0\right)$. The computed insulation is greater than for cylindrical waves, particularly for high frequencies.

\section{REFERENCES}

1. M. H. CYril 1957 Handbook of Noise Control. New York: McGraw-Hill Book Company.

2. A. C. C. WARNOCK and W. FASOLD 1997 Sound Insulation: Airborne and Impact, Encyclopedia of Acoustics, Vol. 3, pp. 1129-1161. New York: Wiley-Interscience Publication.

3. E. C. SEWELL 1970 Journal of Sound and Vibration 12, 21-32. Transmission of reverberant sound through a single-leaf partition surrounded by an infinite rigid baffle.

4. B. H. ShARP 1978 Noise Control Engineering 11, 53-63. Prediction methods for the sound transmission of building elements. 
5. L. CREMER 1942 Akustische Zeitschrift 7, 81-102. Theorie der Shalldämmung dünner Wände bei schrägem Einfall.

6. J. R. Callister, A. R. George and G. E. Freeman 1999 Journal of Sound and Vibration 222, 145-151. An empirical scheme to predict the sound transmission loss of single-thickness panels.

7. I. I. NoviKov 1998 Applied Acoustics 54, 83-90. Low-frequency sound insulation of thin plates.

8. A. Osipov, P. Mees and G. Vermeir 1997 Applied Acoustics 52, 273-288. Low-frequency airborne sound transmission through single partitions in buildings.

9. J. A. Steel and R. J. M. Craik 1994 Journal of Sound and Vibration 178, 553-561. Statistical energy analysis of structure-borne sound transmission by finite element methods.

10. P. Hynnä, P. Klinge and J. VuOKSINEN 1995 Journal of Sound and Vibration 180, 583-607. Prediction of structure-borne sound transmission in large welded ship structures using statistical energy analysis.

11. R. J. M. CraiK and R. S. SMith 2000 Applied Acoustics 61, 223-245. Sound transmission through double leaf lightweight partitions part I: airborne sound.

12. A. Osipov, P. Mees and G. Vermeir 1997 Proceedings of Inter-Noise 2, 759-762. Numerical simulation of airborne sound transmission at low frequencies: the influence of the room and the partition parameters.

13. S. P. S. Maluski and B. M. Gibis 2000 Journal of the Acoustical Society of America 108, 1741-1751. Application of a finite-element to low-frequency sound insulation in dwellings.

14. F. C. Sgard, N. Atalla and J. Nicolas 2000 Journal of Acoustitcal Society of America 108, 2865-2872. A numerical model for the low frequency diffuse field sound transmission loss of double-wall sound barriers with elastic porous linings.

15. M. Tinnsten, M. Jonsson and Ö. Johansson 2001 Acta Acustica 87, 117-127. Prediction and verification of acoustic radiation.

16. A. TAdeu, J. António and L. Godinho Computational Mechanics Journal 27, 484-491. Green's function for two-and-a-half dimensional elastodynamic problems in a half-space.

17. A. TADEU and J. ANTÓNIO Journal of Computer Modeling in Engineering and Sciences, CMES 2, 477-495. 2.5D Green's functions for elastodynamic problems in layered acoustic and elastic formations.

18. L. L. BerAneK and I. L. VÉR 1992 Noise and Vibration Control Engineering. New York: Wiley.

19. K. F. Graff 1975 Wave Motion in Elastic Solids. New York: Dover Publications, Inc.

20. A. J. B. TAdeu and E. KaUsel 2000 Journal of Engineering Mechanics, American Society of Civil Engineers 126, 1093-1097. Green's functions for two-and-a-half dimensional elastodynamic problems.

21. H. A. Pedersen, F. J. Sánchez-Sesma and M. Campillo 1994 Bulletin of the Seismological Society of America 84, 1169-1183. Three-dimensional scattering by two-dimensional topographies.

22. A. S. PApageorgiou and D. Pei 1998 Earthquake Engineering and Structural Dynamics 27, 619-638. A discrete wavenumber boundary element method for study of 3-D response of 2-D scatterers.

23. H. Lamb 1904 Philosophical Transactions of the Royal Society of London A 203, 1-42. On the propagation of tremors at the surface of an elastic solid.

24. M. BOUCHON 1979 Journal of Geophysical Research 84, 3609-3614. Discrete wave number representation of elastic wave fields in three-space dimensions.

25. J. Kim and A. S. PAPAgEORgIOU 1993 Journal of Engineering Mechanics, American Society of Civil Engineers 119, 603-624. Discrete wavenumber boundary element method for 3-D scattering problems.

26. M. BOUCHON, and K. AKI 1977 International Journal of Numerical Methods in Engineering 26, 1709-1728. Time-domain transient elastodynamic analysis of 3D solids by BEM.

27. E. KAUSEL 1992 Journal of Engineering Mechanics, American Society of Civil Engineers 118, 121-734. Frequency domain analysis of undamped systems.

28. I. S. GradshteYn and I. M. RYZHIK 1980 Tables of Integrals, Series, and Products. New York: Academic Press.

\section{APPENDIX A: THE GREEN FUNCTIONS FOR A TWO-AND-A-HALF-DIMENSIONAL FULL SPACE}

\section{A.1. SOLID FORMATION}

Consider an infinite, homogeneous space subjected at point $\left(x_{0}, y_{0}\right)$ to a spatially varying line load of the form $p(x, y, z, t)=\delta\left(x-x_{0}\right) \delta\left(y-y_{0}\right) \mathrm{e}^{\mathrm{i}\left(\omega t-k_{z} z\right)}$ acting in one of the three 
co-ordinate directions. Here, $\delta(x)$ and $\delta(y)$ are Dirac-delta functions, $\omega$ is the frequency of the load and $k_{z}$ is the wavenumber in $z$ (see Figure 1(a)). The response to this load can be calculated by applying a spatial Fourier transform in the $z$ direction to the Helmholtz equations for a point load (see, e.g., reference [28]).

After some mathematical manipulations, a final set of the Green functions can be expressed in terms of the compressional and rotational potentials. These functions can be written as a summation if an infinite number of virtual sources are distributed along the $x$ direction, at equal intervals $L_{x}$. In the case of a sinusoidal harmonic line load along the $z$ direction, acting in the direction of the $y$-axis, a final set of the Green functions can be expressed in terms of the compressional and rotational potentials, $\phi^{y}, \psi_{x}^{y}, \psi_{y}^{y}$ and $\psi_{z}^{y}$, from which the following three components of displacement can be calculated $\left(G_{i j}\right.$ in direction $i$ due to a load applied in direction $j$ )

$$
\begin{gathered}
G_{x y}^{f u l l}=G_{y x}^{f u l l}=E_{a} \sum_{n=-N}^{n=+N}\left[-\mathrm{i} \operatorname{sgn}\left(y-y_{0}\right) k_{n} E_{b}+\mathrm{i} \operatorname{sgn}\left(y-y_{0}\right) k_{n} E_{c}\right] E_{d}, \\
G_{y y}^{f u l l}=E_{a} \sum_{n=-N}^{n=+N}\left[-\mathrm{i} v_{n} E_{b}+\left(\frac{\mathrm{i} v_{z n}^{2}}{\gamma_{n}}\right) E_{c}\right] E_{d}, \\
G_{z y}^{f u l l}=E_{a} \sum_{n=-N}^{n=+N}\left[-\mathrm{i} \operatorname{sgn}\left(y-y_{0}\right) k_{z} E_{b}+\mathrm{i} \operatorname{sgn}\left(y-y_{0}\right) k_{z} E_{c}\right] E_{d},
\end{gathered}
$$

where $E_{a}=1 /\left(2 \rho \omega^{2} L_{x}\right), E_{b}=\mathrm{e}^{-\mathrm{i} v_{n}\left|y-y_{0}\right|}, E_{c}=\mathrm{e}^{-\mathrm{i} \gamma_{n}\left|y-y_{0}\right|}, E_{d}=\mathrm{e}^{-\mathrm{i} k_{n}\left(x-x_{0}\right)}, v_{z n}=\sqrt{-k_{z}^{2}-k_{n}^{2}}$, $v_{n}=\sqrt{k_{p}^{2}-k_{z}^{2}-k_{n}^{2}}$ and $\left(\operatorname{Im}\left(v_{n}\right) \leqslant 0\right), \gamma_{n}=\sqrt{k_{s}^{2}-k_{z}^{2}-k_{n}^{2}}$, with $\left(\operatorname{Im}\left(\gamma_{n}\right) \leqslant 0\right), k_{n}=\left(2 \pi / L_{x}\right) n$, $k_{p}=\omega / \alpha, k_{s}=\omega / \beta, \alpha=\sqrt{(\lambda+2 \mu) / \rho}$ and $\beta=\sqrt{\mu / \rho}$ are the speeds for $P$ (pressure) waves and $S$ (shear) waves, respectively, $\lambda$ and $\mu$ are the Lamé constants, and $\rho$ is the mass density.

Readers can find the complete derivation of these equations in references $[16,20]$.

\section{A.2. FLUID FORMATION}

The Green functions for a sinusoidal pressure line load applied at the point $\left(x_{0}, y_{0}\right)$, in a fluid formation, can be obtained using a process similar to that described above, leading to the expressions

$$
\begin{gathered}
G_{f x}^{f u l l}=-\frac{1}{L_{x}} \sum_{n=-N}^{n=+N}\left[\left(\frac{-\alpha_{f}^{2}}{\omega^{2} \lambda_{f}}\right) \frac{k_{n}}{v_{n}^{f}} E_{f}\right] E_{d}, \\
G_{f y}^{f u l l}=-\frac{1}{L_{x}} \sum_{n=-N}^{n=+N}\left[\left(\frac{-\alpha_{f}^{2}}{\omega^{2} \lambda_{f}}\right) \operatorname{sgn}\left(y-y_{0}\right) E_{f}\right] E_{d}, \\
G_{f z}^{f u l l}=-\frac{1}{L_{x}} \sum_{n=-N}^{n=+N}\left[\left(\frac{-\alpha_{f}^{2}}{\omega^{2} \lambda_{f}}\right) \frac{k_{z}}{v_{n}^{f}} E_{f}\right] E_{d},
\end{gathered}
$$

in which $E_{d}=\mathrm{e}^{-\mathrm{i} k_{n}\left(x-x_{0}\right)}, k_{n}=\left(2 \pi / L_{x}\right) n, v_{n}^{f}=\sqrt{k_{p_{f}}^{2}-k_{z}^{2}-k_{n}^{2}}$ with $\left(\operatorname{Im}\left(v_{n}^{f}\right) \leqslant 0\right), k_{p_{f}}=\omega / \alpha_{f}$, $\alpha_{f}=\sqrt{\lambda_{f} / \rho_{f}}$ is the acoustic (dilatational) wave speed of the fluid, $\lambda_{f}$ is the fluid Lamé 
constant, $\rho_{f}$ is the mass density of the fluid, $E_{f}=\mathrm{e}^{-i v_{n}^{f}\left|y-y_{0}\right|}, v_{n}^{f}=\sqrt{k_{p_{f}}^{2}-k_{z}^{2}-k_{n}^{2}}$ with $\left(\operatorname{Im}\left(v_{n}^{f}\right) \leqslant 0\right), k_{p_{f}}=\omega / \alpha_{f}$.

\section{APPENDIX B: THE GREEN FUNCTIONS IN A SOLID LAYER FORMATION BOUNDED BY TWO FLUID MEDIA}

\section{B.1. SPATIALLY SINUSOIDAL HARMONIC PRESSURE LOAD ACTING IN THE TOP LAYER OF FLUID}

The Green functions for a solid layer formation, with thickness $h$, bounded by two fluid media, can be expressed as the sum of the source terms equal to those in the full-space and the surface terms needed to satisfy the boundary conditions at the two fluid-solid interfaces (continuity of normal displacements and stresses, and null tangential stresses). For this specific problem, the top and bottom interfaces both generate surface terms, which can be expressed in a form similar to that of the source term.

Imposing the eight stated boundary conditions for each value of $n$ thus yields a system of eight equations in the eight unknown constants. Although this procedure is quite straightforward, the details are rather complex, and for this reason they are not given here. The final system of equations is of the form

$$
\left[a_{i j}^{f} i=1,8 ; j=1,8\right]\left[c_{i}^{f} i=1,8\right]=\left[b_{i}^{f} i=1,8\right] .
$$

Definition of $a_{i j}^{f} i=1,8 ; j=1,8$ :

$$
\begin{aligned}
& a_{11}^{f}=-2 v_{n}, \quad a_{12}^{f}=\frac{-k_{n}^{2}}{\gamma_{n}}+\gamma_{n}, \quad a_{13}^{f}=\frac{-k_{z}^{2}}{\gamma_{n}}, \quad a_{14}^{f}=0, \\
& a_{21}^{f}=-2 v_{n}, \quad a_{22}^{f}=\frac{-k_{n}^{2}}{\gamma_{n}}, \quad a_{23}^{f}=\frac{-k_{z}^{2}}{\gamma_{n}}+\gamma_{n}, \quad a_{24}^{f}=0, \\
& a_{31}^{f}=\left(-k_{s}^{2}-2 v_{z n}^{2}\right) a_{32}^{f}=-2 k_{n}^{2}, \quad a_{33}^{f}=-2 k_{z}^{2}, \quad a_{34}^{f}=-i 2 \rho \omega^{2}\left(v_{n}^{f} \mu\right), \\
& a_{41}^{f}=-\mathrm{i} v_{n}, \quad a_{42}^{f}=\frac{-\mathrm{i} k_{n}^{2}}{\gamma_{n}}, \quad a_{43}^{f}=\frac{-\mathrm{i} k_{z}^{2}}{\gamma_{n}}, \quad a_{44}=\frac{-2 \rho \omega^{2}}{k_{p_{f}}^{2} \lambda_{f}}, \\
& a_{15}^{f}=a_{11}^{f} E_{b h}, \quad a_{16}^{f}=a_{12}^{f} E_{c h}, \quad a_{17}^{f}=a_{13}^{f} E_{c h}, \quad a_{18}^{f}=0, \\
& a_{25}^{f}=a_{21}^{f} E_{b h}, \quad a_{26}^{f}=a_{22}^{f} E_{c h}, \quad a_{27}^{f}=a_{23}^{f} E_{c h}, \quad a_{28}^{f}=0, \\
& a_{35}^{f}=-a_{31}^{f} E_{b h}, \quad a_{36}^{f}=-a_{32}^{f} E_{c h}, \quad a_{37}^{f}=-a_{33}^{f} E_{c h}, \quad a_{38}^{f}=0, \\
& a_{45}^{f}=a_{41}^{f} E_{b h}, \quad a_{46}^{f}=a_{42}^{f} E_{c h}, \quad a_{47}^{f}=a_{43}^{f} E_{c h}, \quad a_{48}^{f}=0, \\
& a_{51}^{f}=a_{11}^{f} E_{b h}, \quad a_{52}^{f}=a_{12}^{f} E_{c h}, \quad a_{53}^{f}=a_{13}^{f} E_{c h}, \quad a_{54}^{f}=0, \\
& a_{61}^{f}=a_{21}^{f} E_{b h}, \quad a_{62}^{f}=a_{22}^{f} E_{c h}, \quad a_{63}^{f}=a_{23}^{f} E_{c h}, \quad a_{64}^{f}=0, \\
& a_{71}^{f}=a_{31}^{f} E_{b h}, \quad a_{72}^{f}=a_{32}^{f} E_{c h}, \quad a_{73}^{f}=a_{33}^{f} E_{c h}, \quad a_{74}^{f}=0,
\end{aligned}
$$




$$
\begin{aligned}
& a_{81}^{f}=a_{41}^{f} E_{b h}, \quad a_{82}^{f}=a_{42}^{f} E_{c h}, \quad a_{83}^{f}=a_{43}^{f} E_{c h}, \quad a_{84}^{f}=0, \\
& a_{55}^{f}=a_{11}^{f}, \quad a_{56}^{f}=a_{12}^{f}, \quad a_{57}^{f}=a_{13}^{f}, \quad a_{58}^{f}=0, \\
& a_{65}^{f}=a_{21}^{f}, \quad a_{66}^{f}=a_{22}^{f}, \quad a_{67}^{f}=a_{23}^{f}, \quad a_{68}^{f}=0, \\
& a_{75}^{f}=-a_{31}^{f}, \quad a_{76}^{f}=-a_{32}^{f}, \quad a_{77}^{f}=-a_{33}^{f}, \quad a_{78}^{f}=a_{34}^{f}, \\
& a_{85}^{f}=a_{41}^{f}, \quad a_{86}^{f}=a_{42}^{f}, \quad a_{87}^{f}=a_{43}^{f}, \quad a_{88}^{f}=-a_{44}^{f} .
\end{aligned}
$$

Definition of $c_{i}^{f} i=1,8$ :

$$
\begin{aligned}
& c_{1}^{f}=A_{n}^{f}, \quad c_{2}^{f}=B_{n}^{f}, \quad c_{3}^{f}=C_{n}^{f}, \quad c_{4}^{f}=D_{n}^{f}, \\
& c_{5}^{f}=E_{n}^{f}, \quad c_{6}^{f}=F_{n}^{f}, \quad c_{7}^{f}=G_{n}^{f}, \quad c_{8}^{f}=H_{n}^{f} .
\end{aligned}
$$

Definition of $b_{i}^{f} i=1,8$ :

$b_{1}^{f}=0, \quad b_{2}^{f}=0$,

$b_{3}^{f}=\frac{-\mathrm{i} 2 \rho \omega^{2}}{v_{n}^{f} \mu} E_{f 1}, \quad b_{4}^{f}=\frac{2 \rho \omega^{2}}{k_{p_{f}}^{2} \lambda_{f}} E_{f 1}$,

$b_{5}^{f}=0, \quad b_{6}^{f}=0$.

$b_{7}^{f}=0, \quad b_{8}^{f}=0$

with $\quad E_{b 1}=\mathrm{e}^{-\mathrm{i} v_{n} y_{0}}, \quad E_{c 1}=\mathrm{e}^{-\mathrm{i} \gamma_{n} y_{0}}, \quad v_{z n}=\sqrt{-k_{z}^{2}-k_{n}^{2}}, \quad E_{f 1}=\mathrm{e}^{-\mathrm{i} v_{n}^{f} y_{0}}, \quad E_{b h}=\mathrm{e}^{-\mathrm{i} v_{n} h}$, $E_{c h}=\mathrm{e}^{-\mathrm{i} \gamma_{n} h}$.

Note that, if $k_{z}=0$ is used, the system of equations derived above is reduced to six unknowns, leading to the two-dimensional Green function for plane strain line loads.

The reader can find a detailed derivation of these equations in reference [17]. 